\title{
EXPONENTIAL STABILITY OF A KIND OF STOCHASTIC DELAY DIFFERENCE EQUATIONS
}

\author{
XIAOHUA DING
}

Received 22 February 2006; Accepted 1 May 2006

We present a Razumilchin-type theorem for stochastic delay difference equation, and use it to investigate the mean square exponential stability of a kind of nonautonomous stochastic difference equation which may also be viewed as an approximation of a nonautonomous stochastic delay integrodifferential equations (SDIDEs), and of a difference equation arises from some of the earliest mathematical models of the macroeconomic "trade cycle" with the environmental noise.

Copyright (c) 2006 Xiaohua Ding. This is an open access article distributed under the Creative Commons Attribution License, which permits unrestricted use, distribution, and reproduction in any medium, provided the original work is properly cited.

\section{Introduction}

The problem of stability of stochastic difference equation has been investigated in a number of papers. We refer the readers to [2,3,13-17]. Some results on the asymptotic behavior of the moments were obtained in [18]. But very few results on the Razumilchin-type theorem for stochastic delay difference equation have been published. In this paper, we present a Razumilchin-type theorem for stochastic delay difference equation, and use it to investigate the mean square exponential stability of a kind of nonautonomous stochastic difference equation.

We consider the equation

$$
X_{n+1}=a\left(n, X_{n-m}, \ldots, X_{n}\right)+b\left(n, X_{n-m}, \ldots, X_{n}\right) \triangle \mu_{n}, \quad n \in \mathbb{Z}^{+},
$$

where $a \in \mathbb{R}, b \in \mathbb{R}, a(n, 0, \ldots, 0)=b(n, 0, \ldots, 0)=0, \mathbb{Z}^{+}=\{0,1, \ldots\}$, and $X_{n}=\xi_{n}(n \in I=$ $\{-m,-m+1, \ldots,-1,0\})$ is the initial segment to be $\mathscr{F}_{0}$-measurable. $\triangle \mu_{n}$ are independent $N(0,1)$-distributed Gaussian random variables.

We denote by $\left(\Omega, \mathscr{F}_{F},\left\{\mathscr{F}_{n}\right\}_{n \in \mathbb{N}}, \mathbb{P}\right)$ a complete filtered probability space, where filtration $\left\{\mathscr{F}_{n}\right\}_{n \in \mathbb{N}}$ is naturally generated: $\mathscr{F}_{n+1}=\sigma\left\{\triangle \mu_{i+1}: i=0,1, \ldots, n\right\}$. Among all the sequences $\left\{X_{n}\right\}_{n \in \mathbb{N}}$ of the random variables, we distinguish those for which $X_{n}$ are $\mathscr{F}_{n}$-measurable for all $n \in \mathbb{N}$. 
2 Exponential stability of stochastic difference equations

\section{Main result}

Definition 2.1. The stochastic difference equation (1.1) is said to be $p$ th moment exponentially stable if there are positive constants $\gamma$ and $N$ such that with initial data $\xi_{n}, n \in I$,

$$
E\left|X_{n}\right|^{p} \leq N E\|\xi\|^{p} e^{-\gamma n} \quad \text { on } n \in \mathbb{Z}^{+},
$$

where $\|\xi\|=\max _{n \in I}\left|\xi_{n}\right|$.

Theorem 2.2. Let all $\lambda, p, c_{1}, c_{2}$ be positive numbers and $q>1$, suppose there exists a Lyapunov function $V: I \cup \mathbb{Z}^{+} \times \mathbb{R} \rightarrow \mathbb{R}^{+}$such that

$$
c_{1}|X|^{p} \leq V(t, X) \leq c_{2}|X|^{p} \quad \forall(n, X) \in I \cup \mathbb{Z}^{+} \times \mathbb{R}
$$

and for all $n \in \mathbb{Z}^{+}$,

$$
E \triangle V\left(n, X_{n}\right)=E\left(V\left(n+1, X_{n+1}\right)-V\left(n, X_{n}\right)\right) \leq-\lambda E V\left(n+1, X_{n+1}\right)
$$

if

$$
E V\left(n+s, X_{n+s}\right)<q E V\left(n+1, X_{n+1}\right) \quad \text { on } s \in I \text {. }
$$

Then for all $\xi_{n}, n \in I,\|\xi\|=\max _{n \in I}\left|\xi_{n}\right|$,

$$
E\left|X_{n}\right|^{p} \leq \frac{c_{2}}{c_{1}} E\|\xi\|^{p} e^{-\gamma n} \quad \text { on } n \in \mathbb{Z}^{+},
$$

where $0<\gamma=\min \{\log (1+\lambda), \log q /(1+m)\}$.

Proof. Let

$$
U(n)=\sup _{s \in I}\left\{e^{\gamma(n+s)} E V\left(n+s, X_{n+s}\right)\right\}, \quad n \in \mathbb{Z}^{+} .
$$

For any $n \in \mathbb{Z}^{+}$, we affirm that

$$
\triangle U(n)=U(n+1)-U(n) \leq 0 .
$$

Otherwise, there exists an $n \in \mathbb{Z}^{+}$such that

$$
e^{\gamma(n+1)} E V\left(n+1, X_{n+1}\right)>U(n),
$$

that is,

$$
e^{\gamma(n+1)} E V\left(n+1, X_{n+1}\right)>e^{\gamma(n+s)} E V\left(n+s, X_{n+s}\right), \quad s \in I .
$$

For any $s \in I$, the inequality (2.4) implies

$$
E V\left(n+s, X_{n+s}\right)<e^{\gamma(m+1)} E V\left(n+1, X_{n+1}\right)<q E V\left(n+1, X_{n+1}\right)
$$


since $\gamma<\log q /(1+m)$. Thus, by condition $(2.3)$,

$$
E \triangle V\left(n, X_{n}\right)=E\left(V\left(n+1, X_{n+1}\right)-V\left(n, X_{n}\right)\right) \leq-\lambda E V\left(n+1, X_{n+1}\right)
$$

We obtain that

$$
E V\left(n+1, X_{n+1}\right) \leq \frac{1}{1+\lambda} E V\left(n, X_{n}\right)
$$

Multiplying both sides of above inequality by $e^{\gamma(n+1)}$ and noting that $\gamma \leq \log (1+\lambda)$, we can get

$$
e^{\gamma(n+1)} E V\left(n+1, X_{n+1}\right) \leq e^{\gamma(n+1)} \frac{1}{1+\lambda} E V\left(n, X_{n}\right) \leq e^{\gamma n} E V\left(n, X_{n}\right)
$$

From the definition of $U(n)$, we have

$$
e^{\gamma(n+1)} E V\left(n+1, X_{n+1}\right) \leq U(n) .
$$

It is a contradiction with assumption (2.8), therefore, (2.7) holds. Thus, it follows from (2.7) immediately that

$$
U(n) \leq U(0), \quad \forall n \in \mathbb{Z}^{+}
$$

From the definition of $U(n)$ and the condition (2.2), we obtain that the inequality (2.5) holds.

\section{Application}

3.1. A numerical approximation to SDIDEs. We consider the stochastic delay difference equation

$$
\begin{gathered}
X_{n+1}=X_{n}+h\left[a(n) X_{n}+h \sum_{i=1}^{m} K(n, i) X_{n-i}\right]+\sqrt{h} c(n) X_{n-m} \Delta \mu_{n}, \quad n \in \mathbb{Z}^{+}, \\
X_{n}=\xi_{n}, \quad n \in I,
\end{gathered}
$$

where $h>0$ is a nonrandom parameter. For the functions $a(\cdot), b(\cdot)$, and $K(\cdot, \cdot)$, suppose that

$$
\begin{gathered}
A(m, n, h)=2 a(n)+a^{2}(n) h+m|1+a(n) h| h^{2}, \\
B(m, n, h)=c^{2}(n)+h\left(m h^{2}+|1+a(n) h|\right) \sum_{i=1}^{m} K^{2}(n, i)
\end{gathered}
$$


satisfy the conditions $(\mathrm{H} 1)$ and $(\mathrm{H} 2)$ as the following:

(H1) $A(m, n, h)+B(m, n, h) \leq-\delta(m, h)<0$ for $n \in \mathbb{Z}^{+}$;

(H2) $-1<h \mu(m, h) \leq h A(m, n, h)$.

Let

$$
\begin{gathered}
q(m, h)=\inf _{n \in \mathbb{Z}^{+}}\left\{\frac{B(m, n h)-A(m, n, h)}{2 B(m, n, h)}\right\}, \\
\lambda(m, h)=-\inf _{n \in \mathbb{Z}^{+}}\left\{\frac{A(m, n, h)+B(m, n, h)}{2(1+h A(m, n, h))}\right\} .
\end{gathered}
$$

From the conditions (H1) and (H2), we know that

$$
q(m, h)>1, \quad \lambda(m, h)>0 .
$$

Equation (3.1) may also be viewed as an approximation of the stochastic delay integrodifferential equation

$$
\begin{gathered}
d X(t)=\left[a(t) X(t)+\int_{t-\tau}^{t} K(t, t-\theta) X(\theta) d \theta\right] d t+c(t) X(t-\tau) d W(t), \quad t>0, \\
X(t)=\xi(t), \quad t \in[-\tau, 0],
\end{gathered}
$$

where $W(t)$ is a standard Brownian motion. Here, setting $h=\tau / m$ and approximating the differential part of (3.5) with the Euler-Maruyama method and the integral part with composite left-side rectangle rule [12], $t_{n}=n h$, write $X_{n}$ for an approximate value to $X(n h)$, and use $X_{n-m}$ to approximate the delayed argument $X\left(t_{n}-\tau\right)$. When $n \in$ $I=\{-m,-m+1, \ldots,-1,0\}$, we have $X_{n}=\xi\left(t_{n}\right)$. Moreover, the increments $\sqrt{h} \Delta \mu_{n}:=$ $W\left(t_{n+1}\right)-W\left(t_{n}\right)$ are independent $N(0, h)$-distributed Gaussian random variables, so $\triangle \mu_{n}$ are independent $N(0,1)$-distributed Gaussian random variables. We assume $X_{n}$ to be $\mathscr{F}_{n^{-}}$ measurable at the mesh-points $t_{n}$. It is therefore to be hoped for $h$ sufficiently small that solutions of (3.1) have similar asymptotic properties to those of (3.5). A statement of these asymptotic results for stochastic delay differential equations can be found in, for example, $[1,4-11]$.

Here, we use the above Razumilchin-type Theorem 2.2 to study the moment exponential stability of (3.1).

Theorem 3.1. Assume conditions (H1) and (H2) are satisfied, the solution sequence $\left\{X_{n}\right\}$ produced by the difference equation (3.1) satisfies

$$
E\left|X_{n}\right|^{2} \leq E\|\xi\|^{2} e^{-\gamma(m, h) n h} \quad \text { on } n \in \mathbb{Z}^{+}
$$

where $0<\gamma(m, h)=\min \{\log (1+h \lambda(m, h)) / h, \log q(m, h) / h(1+m)\}$. 
Proof. Define a Lyapunov function $V(n, X)=|X|^{2}$. Clearly, the condition (2.2) in Theorem 2.2 is satisfied naturally with $c_{1}=c_{2}=1, p=2$. We see from (3.1) that

$$
\begin{aligned}
X_{n+1}^{2}= & (1+a(n) h)^{2} X_{n}^{2}+h^{4}\left(\sum_{i=1}^{m} K(n, i) X_{n-i}\right)^{2}+\left(\sqrt{h} c(n) \triangle \mu_{n}\right)^{2} X_{n-m}^{2} \\
& +2(1+a(n) h) h^{2} \sum_{i=1}^{m} K(n, i) X_{n-i} X_{n}+2(1+a(n) h) \sqrt{h} c(n) \triangle \mu_{n} X_{n} X_{n-m} \\
& +2 h^{2} \sqrt{h} c(n) \triangle \mu_{n} \sum_{i=1}^{m} K(n, i) X_{n-i} X_{n-m} \\
\leq & (1+a(n) h)^{2} X_{n}^{2}+m h^{4} \sum_{i=1}^{m} K^{2}(n, i) X_{n-i}^{2}+\left(\sqrt{h} c(n) \triangle \mu_{n}\right)^{2} X_{n-m}^{2} \\
& +|1+a(n) h| h^{2} \sum_{i=1}^{m}\left(K^{2}(n, i) X_{n-i}^{2}+X_{n}^{2}\right)+2(1+a(n) h) \sqrt{h} c(n) \triangle \mu_{n} X_{n} X_{n-m} \\
& +2 h^{2} \sqrt{h} c(n) \triangle \mu_{n} \sum_{i=1}^{m} K(n, i) X_{n-i} X_{n-m} \\
= & {\left[(1+a(n) h)^{2}+m|1+a(n) h| h^{2}\right] X_{n}^{2} } \\
& +\left(\sqrt{h} c(n) \triangle \mu_{n}\right)^{2} X_{n-m}^{2}+\sum_{i=1}^{m}\left[m h^{4}+|1+a(n) h| h^{2}\right] K^{2}(n, i) X_{n-i}^{2} \\
& +2(1+a(n) h) \sqrt{h} c(n) \triangle \mu_{n} X_{n} X_{n-m}+2 h^{2} \sqrt{h} c(n) \triangle \mu_{n} \sum_{i=1}^{m} K(n, i) X_{n-i} X_{n-m} . \\
& +2(n)
\end{aligned}
$$

Note that $E\left(\triangle \mu_{n}\right)=0, E\left[\left(\triangle \mu_{n}\right)^{2}\right]=1$ and $X_{n}, X_{n-k}$ are $\mathscr{F}_{n}$-measurable, Hence

$$
\begin{gathered}
E\left(\triangle \mu_{n} X_{n-k} X_{n-m}\right)=E\left(X_{n-k} X_{n-m} E\left(\triangle \mu_{n} \mid \mathscr{F}_{n}\right)\right)=0, \\
E\left(\triangle \mu_{n} X_{n-m}^{2}\right)=E\left(X_{n-m}^{2} E\left(\triangle \mu_{n} \mid \mathscr{F}_{n}\right)\right)=0, \\
E\left(\triangle \mu_{n}^{2} X_{n-m}^{2}\right)=E\left(X_{n-m}^{2} E\left(\triangle \mu_{n}^{2} \mid \mathscr{F}_{n}\right)\right)=E\left(X_{n-m}^{2}\right) .
\end{gathered}
$$

From (3.7), (3.8) we get that

$$
\begin{aligned}
E V\left(n+1, X_{n+1}\right) \leq & {\left[(1+a(n) h)^{2}+m|1+a(n) h| h^{2}\right] E V\left(n, X_{n}\right) } \\
& +(\sqrt{h} c(n))^{2} E V\left(n-m, X_{n-m}\right) \\
& +h^{2}\left(m h^{2}+|1+a(n) h|\right) \sum_{i=1}^{m} K^{2}(n, i) E V\left(n-i, X_{n-i}\right) .
\end{aligned}
$$


6 Exponential stability of stochastic difference equations

Let $q=q(m, h)$, from assumption (2.4), we also have

$$
\begin{aligned}
E V\left(n+1, X_{n+1}\right) \leq & {\left[(1+a(n) h)^{2}+m|1+a(n) h| h^{2}\right] E V\left(n, X_{n}\right) } \\
& +h q(m, h)\left[c^{2}(n)+h\left(m h^{2}+|1+a(n) h|\right) \sum_{i=1}^{m} K^{2}(n, i)\right] E V\left(n+1, X_{n+1}\right) \\
= & (1+h A(m, n, h)) E V\left(n, X_{n}\right)+h q(m, h) B(m, n, h) E V\left(n+1, X_{n+1}\right) \\
\leq & (1+h A(m, n, h)) E V\left(n, X_{n}\right)+h \frac{B(m, n, h)-A(m, n, h)}{2} E V\left(n+1, X_{n+1}\right),
\end{aligned}
$$

that is,

$$
E \triangle V\left(n, X_{n}\right) \leq h \frac{B(m, n, h)-A(m, n, h)}{2(1+h A(m, n, h))} E V\left(n+1, X_{n+1}\right) .
$$

By the definition of $\lambda(m, h)$ and (3.4), we get

$$
E \triangle V\left(n, X_{n}\right) \leq-h \lambda(m, h) E V\left(n+1, X_{n+1}\right) .
$$

Therefore, the inequality (3.6) holds by Theorem 2.2.

3.2. Models of macroeconomics. Consider the following nonlinear delay difference equation:

$$
x(n+1)=c x(n)+f(x(n)-x(n-m))+\varepsilon x(n) \triangle \mu_{n},
$$

where $c \in[0,1)$ and $\varepsilon$ are constants, $m$ is a positive integer. $\triangle \mu_{n}$ are independent $N(0,1)$ distributed Gaussian random variables. We assume that $x(n)$ are $\mathscr{F}_{n}$-measurable for all $n \in \mathbb{N}$, and we have $x(n)=\xi_{n}$ when $n \in I . f: \mathbb{R} \rightarrow \mathbb{R}$ satisfies $f(0)=0, f(u) \neq 0$ for $u \neq 0$, and there exists a constant $\alpha$ such that

$$
|f(u)| \leq \alpha|u|
$$

Such equation arises from some of the earliest mathematical models of the macroeconomic "trade cycle" with the environmental noise.

Theorem 3.2. Assume that the conditions (3.14) and

$$
0 \leq c<-2 \alpha+\sqrt{2 \alpha^{2}-\varepsilon^{2}-2 \alpha+1}
$$

are satisfied. Then there exists positive constants $\gamma$ such that with initial data $\xi_{n}, n \in I$,

$$
E|X(n)|^{2} \leq E\|\xi\|^{2} e^{-\gamma n} \quad \text { on } n \in \mathbb{Z}^{+} .
$$


Proof. Also define a Lyapunov function $V(n, x)=|x|^{2}$. Similar to the proof of Theorem 3.1 , let

$$
\begin{aligned}
& q=\frac{1-c^{2}-2 \alpha c-2 \alpha^{2}-\varepsilon^{2}+2 \alpha}{2 \alpha(2+c)}, \\
& \lambda=\frac{[2(2+c) \alpha-1]\left(c^{2}+2 \alpha^{2}+\varepsilon^{2}+3 c \alpha\right)+1}{2(2+c) \alpha\left(c^{2}+2 \alpha^{2}+\varepsilon^{2}+3 c \alpha\right)},
\end{aligned}
$$

the inequality (3.16) with $\gamma=\min \{\log (1+\lambda), \log q /(1+k)\}$ can be completed by Theorem 2.2 easily.

\section{Examples}

4.1. A numerical approximation to autonomous SDIDEs. Consider the autonomous stochastic delay integrodifferential equation as (3.5),

$$
\begin{gathered}
d X(t)=\left[a X(t)+b \int_{t-\tau}^{t} X(\theta) d \theta\right] d t+\sqrt{h} c X(t-\tau) d W(t), \quad t>0, \\
X(t)=\xi(t), \quad t \in[-\tau, 0] .
\end{gathered}
$$

Approximating the differential part of (3.5) with the Euler-Maruyama method and the integral part with composite left-side rectangle rule, we get the difference equation as follows:

$$
X_{n+1}=X_{n}+\left(a X_{n}+b h \sum_{k=1}^{m} X_{n-k}\right) h+\sqrt{h} c X_{n-m} \triangle \mu_{n}, \quad n \in \mathbb{Z}^{+} .
$$

Here, $h=\tau / m$ and

$$
\begin{aligned}
& A(m, n, h)=2 a+a^{2} h+\tau h|1+a h|, \\
& B(m, n, h)=c^{2}+b^{2} \tau(\tau h+|1+a h|) .
\end{aligned}
$$

Since

$$
1+h A(m, n, h)=(1+a h)^{2}+\tau h|1+a h|>0,
$$

so that the (H2) is satisfied, and if

$$
2 a+c^{2}+b^{2} \tau+\left(a^{2}+\tau+b^{2} \tau^{2}-a b^{2} \tau\right) h-a \tau h^{2}<0,
$$

then $(\mathrm{H} 1)$ also is satisfied.

By letting

$$
\begin{aligned}
q(m, h) & =\frac{B(m, n h)-A(m, n, h)}{2 B(m, n, h)}, \\
\lambda(m, h) & =-\frac{A(m, n, h)+B(m, n, h)}{2(1+h A(m, n, h))} .
\end{aligned}
$$

From Theorem 3.1 we know that the inequality (3.6) holds. 
8 Exponential stability of stochastic difference equations

From the above analysis and (4.5), we can get the theorem as follows.

Theorem 4.1. Assume condition $2 a+c^{2}+b^{2} \tau<0$ is satisfied, then there exists an $h^{*}>0$ such that for all $0<h<h^{*}$, the solution sequence $\left\{X_{n}\right\}$ produced by (4.2) satisfies

$$
E\left|X_{n}\right|^{2} \leq E\|\xi\|^{2} e^{-\gamma(m, h) n h} \quad \text { on } n \in \mathbb{Z}^{+},
$$

where $0<\gamma(h)=\min \{\log (1+h \lambda(h)) / h, \log q(h) /(h+\tau)\}$, and

$$
h^{*}=\frac{a^{2}+\tau+b^{2} \tau^{2}-a b^{2} \tau-\sqrt{\left(a^{2}+\tau+b^{2} \tau^{2}-a b^{2} \tau\right)^{2}+a \tau\left(2 a+c^{2}+b^{2} \tau\right)}}{2 a \tau} .
$$

Corollary 4.2. Assume condition $2 a+c^{2}+b^{2} \tau<0$ is satisfied, then for any given $\varepsilon>0$, there exists an $h(\epsilon)>0$ such that for all $0<h<h(\epsilon)$, the numerical solution sequence $\left\{X_{n}\right\}$ produced by the numerical scheme (4.2) satisfies

$$
E\left|X_{n}\right|^{2} \leq E\|\xi\|^{2} e^{-(\gamma-\epsilon) n h} \quad \text { on } n \in \mathbb{Z}^{+}
$$

where $\gamma=\min \{\lambda, \log q / \tau\}$ with $\lambda=-\left(a+(1 / 2)\left(c^{2}+b^{2} \tau\right)\right)$ and $q=1 / 2-a /\left(c^{2}+b^{2} \tau\right)$.

Proof. From Theorem 4.1, the corollary is a consequence of the fact that

$$
\begin{aligned}
& \frac{\log (1+h \lambda(h))}{h}=\lambda+O(h), \\
& \frac{\log q(h)}{(h+\tau)}=\frac{\log q}{\tau}+O(h) .
\end{aligned}
$$

4.2. An example of macroeconomics models. Consider the difference equation

$$
x(n+1)=c x(n)+0.25 \sin (x(n)-x(n-3))+0.25 x(n) \triangle \mu_{n},
$$

we can get that if the condition

$$
0 \leq c \leq 0.25
$$

is satisfied, then the inequality (3.6) holds, with $\gamma=\min \{\log (1+\lambda), \log q / 4\}$. Here

$$
\begin{aligned}
& \lambda=\frac{21 / 8-2 c^{2}-c}{2+c} \\
& q=\frac{2+c\left(c^{2}+(3 / 4) c+3 / 16\right)}{(2+c)\left(c^{2}+(3 / 4) c+3 / 16\right)} .
\end{aligned}
$$

\section{Acknowledgment}

This work is supported by the NSF of China (no.10271036) and of HIT(200518). 


\section{References}

[1] J. A. D. Appleby, X. Mao, and A. Rodkina, On stochastic stabilization of difference equations, Discrete and Continuous Dynamical Systems 15 (2006), no. 3, 843-857.

[2] V. Kolmanovskii and L. Shaikhet, General method of Lyapunov functionals construction for stability investigation of stochastic difference equations, Dynamical Systems and Applications, World Sci. Ser. Appl. Anal., vol. 4, World Scientific, New Jersey, 1995, pp. 397-439.

[3] - Some peculiarities of the general method of Lyapunov functionals construction, Applied Mathematics Letters 15 (2002), no. 3, 355-360.

[4] X. X. Liao and X. Mao, Almost sure exponential stability of neutral differential difference equations with damped stochastic perturbations, Electronic Journal of Probability 1 (1996), no. 8, 1-16.

[5] K. Liu and X. Mao, Exponential stability of non-linear stochastic evolution equations, Stochastic Processes and Their Applications 78 (1998), no. 2, 173-193.

[6] X. Mao, Exponential stability in mean square for stochastic differential equations, Stochastic Analysis and Applications 8 (1990), no. 1, 91-103.

[7] _ Exponential Stability of Stochastic Differential Equations, Monographs and Textbooks in Pure and Applied Mathematics, vol. 182, Marcel Dekker, New York, 1994.

[8] _ Razumikhin-type theorems on exponential stability of stochastic functional-differential equations, Stochastic Processes and Their Applications 65 (1996), no. 2, 233-250.

[9] X. Mao and A. Shah, Exponential stability of stochastic differential delay equations, Stochastics and Stochastics Reports 60 (1997), no. 1-2, 135-153.

[10] V. J. Mizel and V. Trutzer, Stochastic hereditary equations: existence and asymptotic stability, Journal of Integral Equations 7 (1984), no. 1, 1-72.

[11] S. E. A. Mohammed, Stochastic Functional Differential Equations, Research Notes in Mathematics, vol. 99, Pitman, Massachusetts, 1984.

[12] V. D. Potapov, Numerical method for investigation of stability of stochastic integro-differential equations, Applied Numerical Mathematics 24 (1997), no. 2-3, 191-201.

[13] A. Rodkina, On asymptotic behaviour of solutions of stochastic difference equations, Nonlinear Analysis 47 (2001), no. 7, 4719-4730.

[14] A. Rodkina and G. Berkolaiko, On asymptotic behavior of solutions to linear discrete stochastic equation, Proceedings of the International Conference "2004-Dynamical Systems and Applications", Antalya, July 2004, pp. 614-623.

[15] A. Rodkina and X. Mao, On boundedness and stability of solutions of nonlinear difference equation with nonmartingale type noise, Journal of Difference Equations and Applications 7 (2001), no. 4 , $529-550$.

[16] A. Rodkina, X. Mao, and V. Kolmanovskii, On asymptotic behaviour of solutions of stochastic difference equations with Volterra type main term, Stochastic Analysis and Applications 18 (2000), no. $5,837-857$.

[17] A. Rodkina and H. Schurz, Global asymptotic stability of solutions of cubic stochastic difference equations, Advances in Difference Equations 2004 (2004), no. 3, 249-260.

[18] H. Schurz, Moment attractivity, stability and contractivity exponents of stochastic dynamical systems, Discrete and Continuous Dynamical Systems 7 (2001), no. 3, 487-515.

Xiaohua Ding: Department of Mathematics, Harbin Institute of Technology (Weihai),

Weihai 264209, China

E-mail address: mathdxh@126.com 


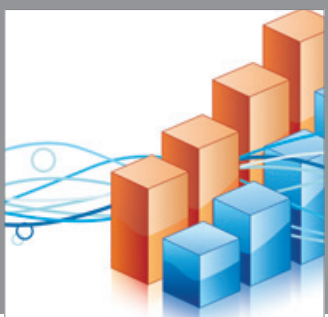

Advances in

Operations Research

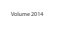

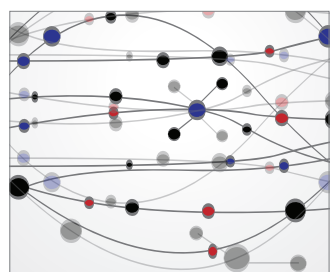

\section{The Scientific} World Journal
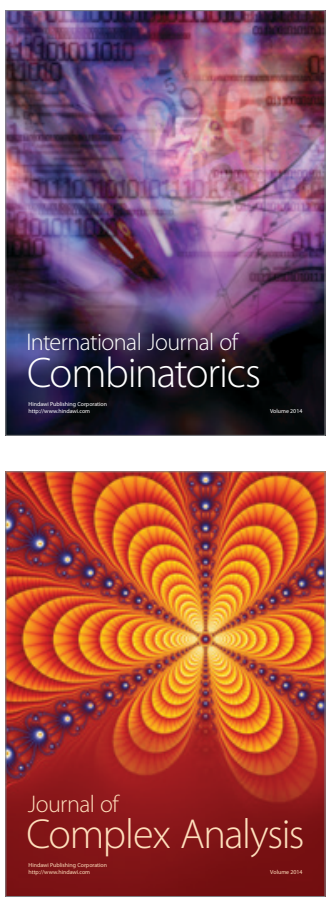

International Journal of

Mathematics and

Mathematical

Sciences
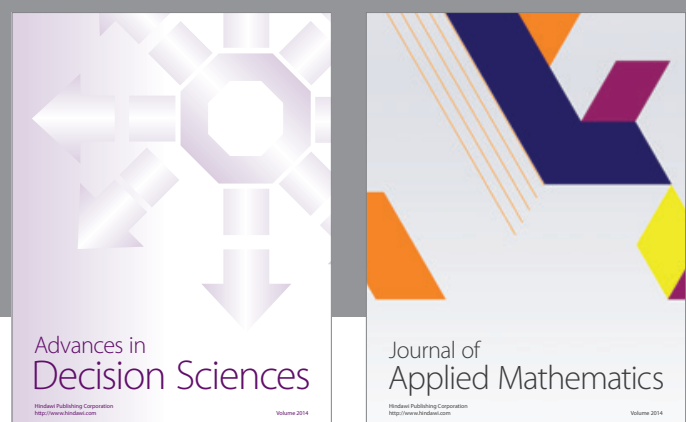

Journal of

Applied Mathematics
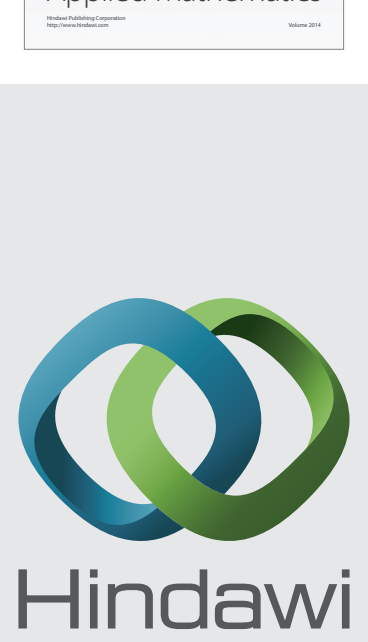

Submit your manuscripts at http://www.hindawi.com
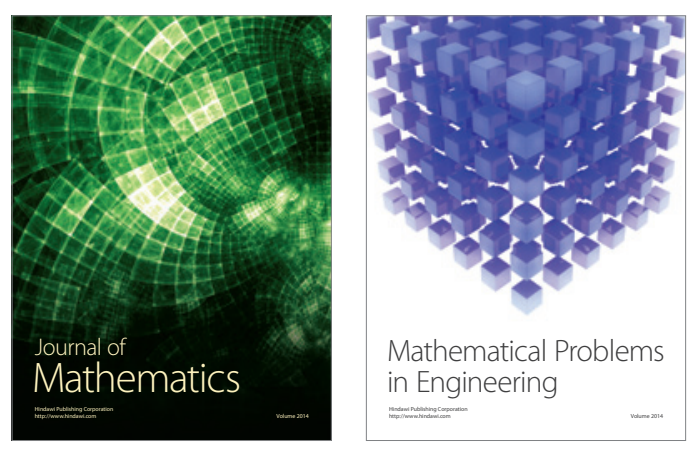

Mathematical Problems in Engineering
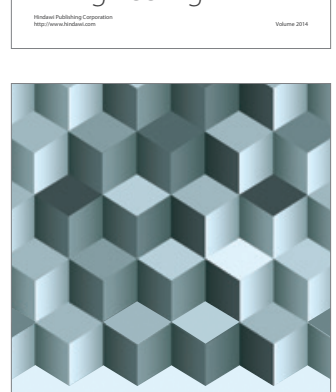

Journal of

Function Spaces
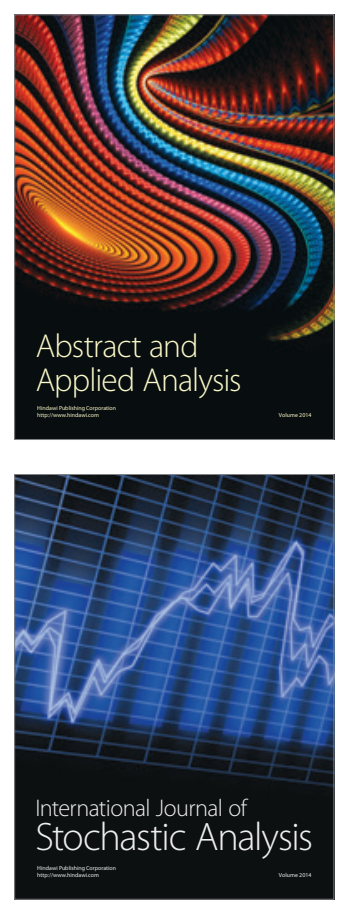

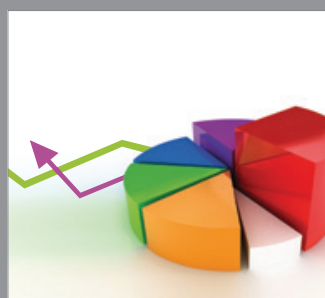

ournal of

Probability and Statistics

Promensencen
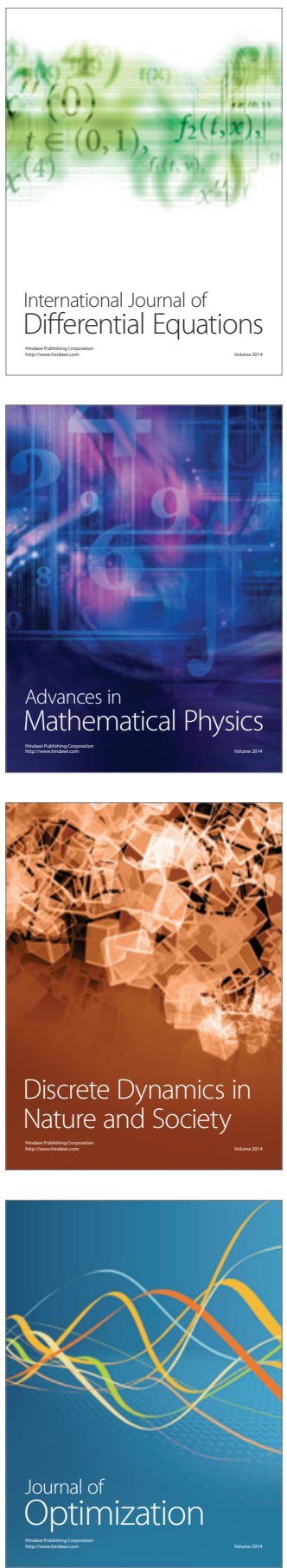